\title{
Resistencia bacteriana en Chile
}

PATRICIA GARCÍA C.

\section{Bacterial resistance to antimicrobial agents}

Bacterial antimicrobial resistance is a complex phenomenon in which several factors as use and abuse of antimicrobial agents, weakening of infection control programs and the existence of multi invaded severely ill patients are involved. As the main consequence of bacterial resistance are antibiotic treatment failure, the enhancement of morbidity and mortality and increasing costs of medical support, it is necessary to control the problem. The initial step of containment is to know the magnitude of bacterial resistance. In our country, obtaining figures has been difficult because of the absence of a unified national surveillance program, with high coverage and a certified quality standard. With the available data at the present obtained of diverse sources of information, the emergence of resistance in Staphylococcus aureus to methicillin, in Streptococcus pneumoniae to penicillin, in Escherichia coli y Klebsiella pneumoniae to third generation cephalosporins and in Pseudomonas aeruginosa to imipenem is concerning. The increasing resistance of $E$. coli and $K$. pneumoniae to third generation cephalosporins due to extended spectrum betalactamases synthesis is also concerning, because they confer resistance to all betalactam antibiotics and their confirmatory tests are scarcely implemented in our country. Pseudomonas aeruginosa resistance to carbapenems is still not a great magnitude problem in Chile, but hospitals with high complexity wards must dispose alternative drugs and methods to confirm the resistance to these antimicrobial agent. In conclusion, the absolute necessity to know in a representative and certified way the magnitude of resistance to the different antibiotic groups is evident, so as to take the necessary therapeutic measures.

Key words: Bacterial resistance; Surveillance; Community acquired infections; Nosocomial infections.

\section{Introducción}

La resistencia bacteriana es un fenómeno biológico natural, de modo que cada vez que se pone en uso un nuevo agente antimicrobiano (AAM) en la práctica clínica, el laboratorio de microbiología detecta cepas resistentes.

Una cepa resistente se define como aquella que es capaz de multiplicarse en presencia de concentraciones mayores que las alcanzadas con dosis terapéuticas.

En general, todos los mecanismos de resistencia pre-existen o se modifican en la naturaleza, ya sea por transferencia de genes de resistencia o por mutaciones, que pueden localizarse en el cromosoma bacteriano o en plasmidios. Por esto se puede suponer que los AAM tendrán actividad por un tiempo limitado, según la presión selectiva que este AAM ejerza sobre la población bacteriana. La presión selectiva resulta de la administración de un AAM que inhibe el crecimiento de microorganismo susceptibles pero selecciona cepas resistentes (naturales o adquiridas) al AAM.

En la Figura 1 se puede observar que el fenómeno de la resistencia bacteriana en muy dinámico: tiene múltiples causas donde la más importante ha sido el uso y abuso de los AAM; sin embargo, el relajamiento en la prácticas de control de infecciones, el aumento del uso de dispositivos y procedimientos médicos invasores y hospederos más susceptibles también han jugado un rol importante en el último tiempo ${ }^{1}$. La consecuencia más importante de la resistencia bacteriana es el fracaso de la terapia antimicrobiana con el consiguiente aumento de la morbi-mortalidad y aumento en los costos. En los Estados Unidos de América se calcula un gasto anual como consecuencia de la resistencia bacteriana, de aproximadamente 4 billones de dólares ${ }^{1}$. Por otra parte, para poder contener el problema y

Laboratorio de Microbiología, Unidad Docente Asociada de Laboratorios Clínicos, Escuela de Medicina, Pontificia

Universidad Católica de Chile.

E-mail: pgarcia@med.puc.cl 
Tabla 1. Fuente de obtención de los datos de resistencia en Chile

\section{Fuente}

Instituto de Salud Pública de Chile MINSAL

\section{Descripción}

Vigilancia de resistencia de patógenos ambulatorios

Metodología centralizada con derivación de cepas al ISP desde los laboratorios locales Calidad de los resultados certificada Representatividad dependiente del envío activo desde los laboratorios locales

Programa de Control de Infecciones MINSAL

\section{Vigilancia de resistencia de patógenos hospita- larios \\ Metodología descentralizada con derivación de datos al MINSAL, obtenidos por los comités de infecciones locales \\ Se desconoce control de calidad realizado por laboratorios locales \\ Alta representatividad}

\section{Red PRONARES}

Vigilancia de resistencia de patógenos ambulatorios y hospitalarios

Metodología descentralizada con derivación de datos desde el laboratorio por sistema WHONET

Calidad certificada por control de calidad local. Poca representatividad del país: 11 centros y sólo 5 en regiones

Datos de Red SENTRY

Vigilancia de resistencia por síndrome clínico de patógenos ambulatorios y hospitalarios Metodología centralizada en Laboratorios de Referencia (EUA)

Derivación de cepas desde sólo 2 centros en Chile Calidad de los resultados certificada Muy escasa representatividad

Publicaciones de datos locales

Publicaciones en revistas chilenas Metodología definida por cada autor Sólo muestra datos de centros individuales

Encuestas

Metodología retrospectiva desde los laboratorios

En general sin certificación de calidad

Baja representatividad evitar las consecuencias, el uso prudente de los AAM y el adecuado control en la infecciones intrahospitalarias parecen ser las mejores herramientas de combate contra la diseminación de la resistencia bacteriana.

Es reconocida como una medida importante para evitar el uso u abuso de los AAM la regulación de la venta de AAM. En Chile existe esta regulación desde septiembre del año 1999; sin embargo, para poder estimar este impacto es necesario conocer la magnitud del problema en Chile.

\section{Magnitud del problema en Chile}

La obtención de datos de resistencia bacteriana en nuestro país, en general no ha sido fácil (Tabla 1).

- El Instituto de Salud Pública de Chile lleva una vigilancia en patógenos asociados a infecciones ambulatorias: Streptococcus pneumoniae, Haemophilus influenzae (como agentes causales de infecciones invasoras), Neisseria sp, Salmonella sp y Shigella. sp. Sin embargo, la 


\section{RESISTENCIA BACTERIANA}

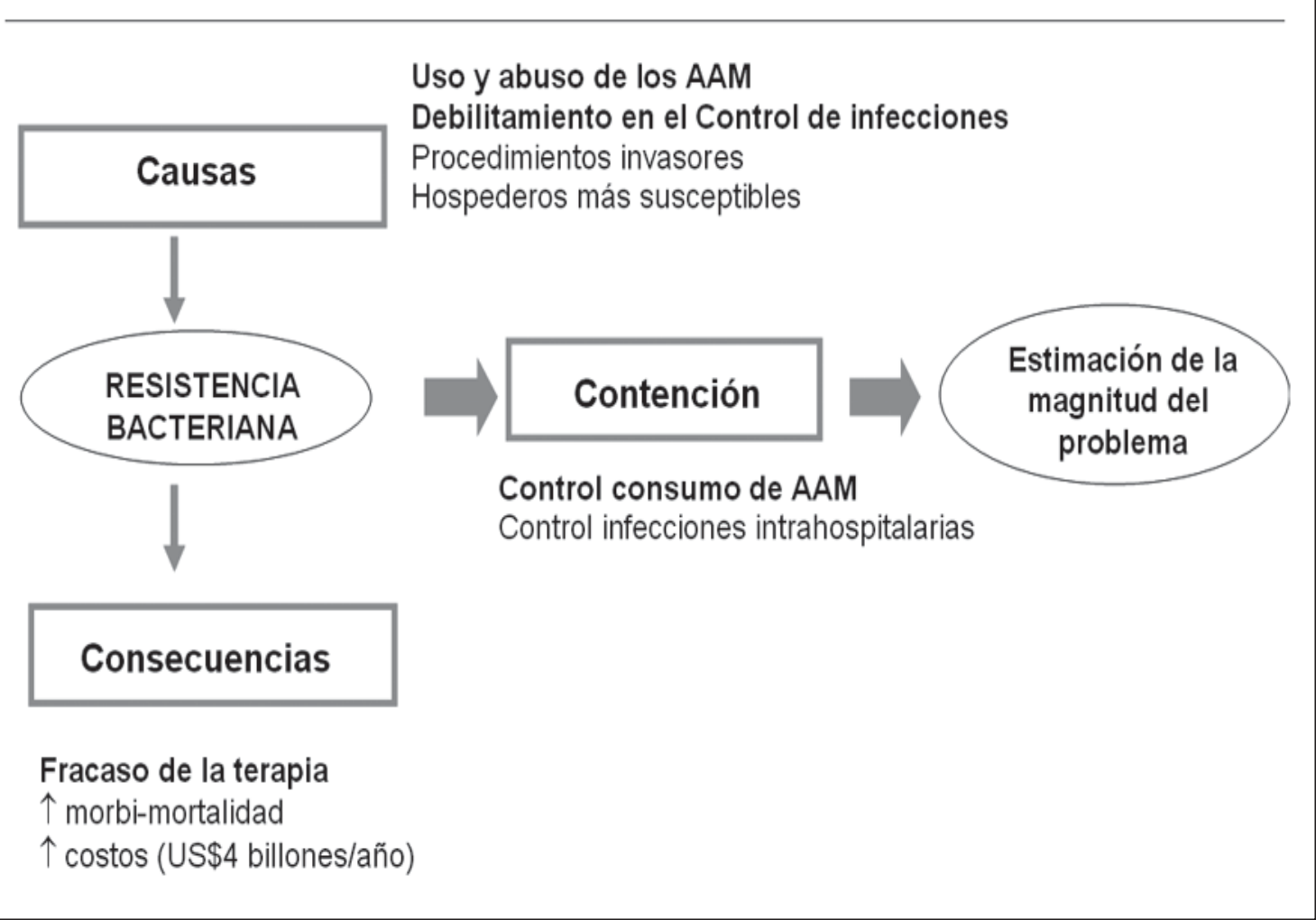

Figura 1. Dinámica del fenómeno de la resistencia bacteriana.

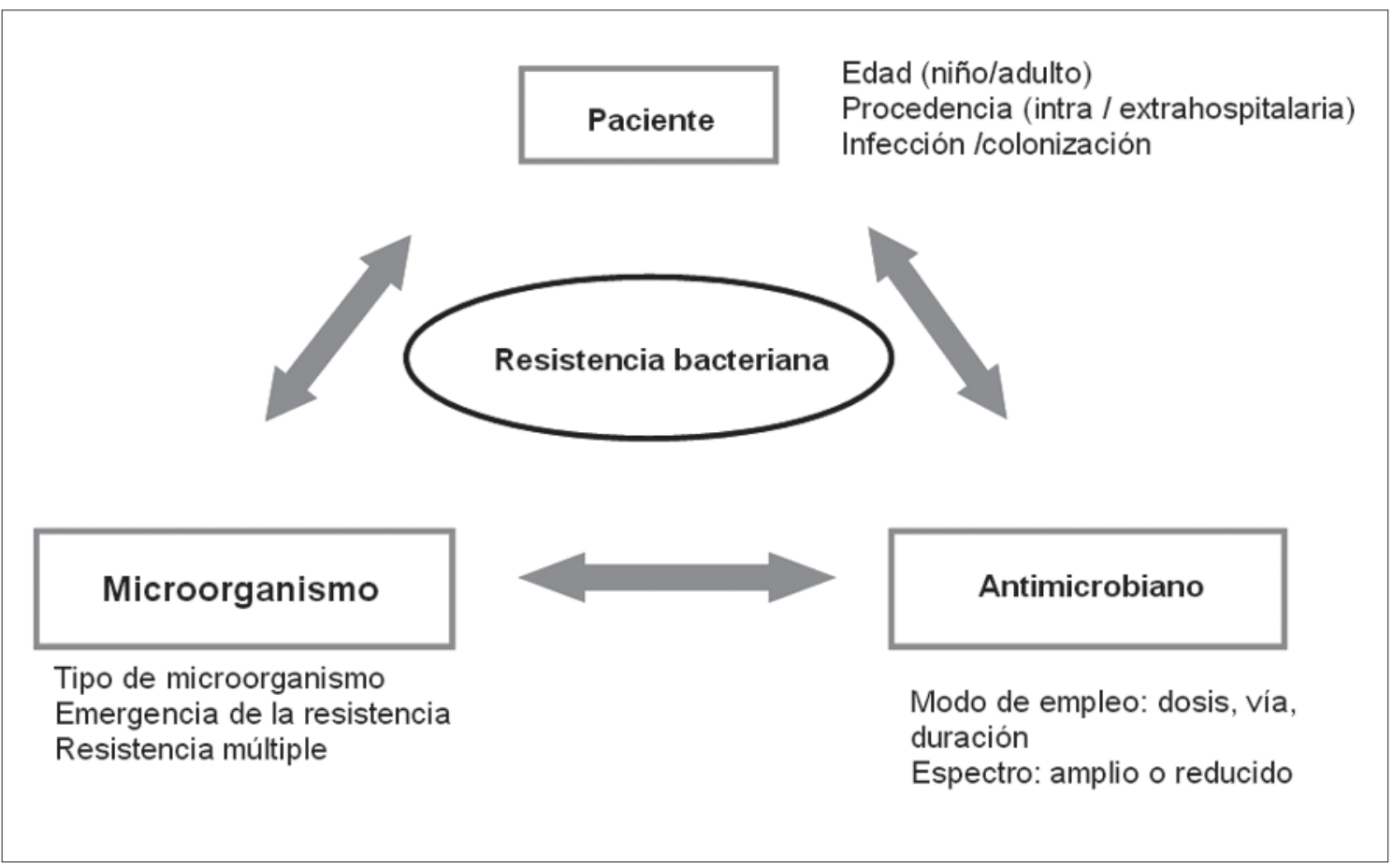

Figura 2. Factores que influyen en la resistencia bacteriana. 


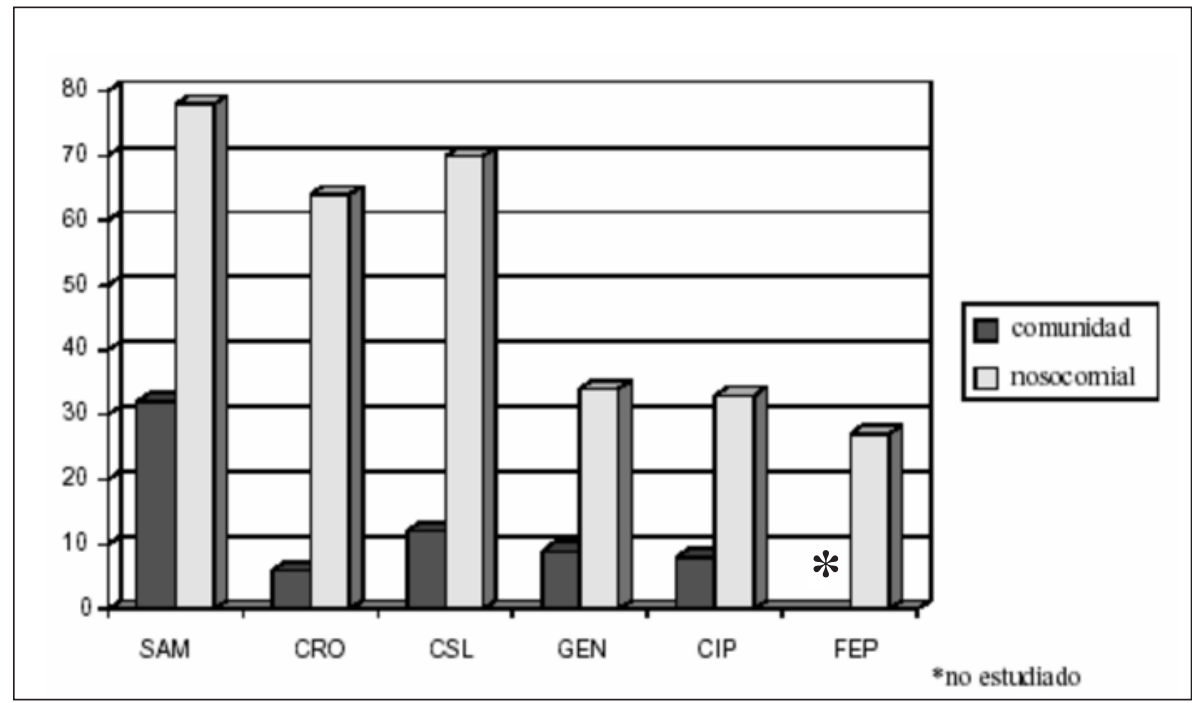

Gráfico 1. Porcentaje de resistencia de Klebsiella spp de la comunidad (122) y nosocomial (100), para ampicilina/sulbactam (SAM), ceftriaxona (CRO), cefoperazona/sulbactam (CSL), gentamicina (GEN), ciprofloxacina (CIP) y cefepime (FEP). Fuente: Pronares; Trucco et al. Rev Chil Infec 2002; 19: S140-8.

metodología centralizada de esta vigilancia (derivación de las cepas al ISP) la hace ser limitada, ya que requiere de una gran inversión de recursos en el nivel central y es difícil lograr buenas coberturas.

- El Ministerio de Salud mediante su Programa de Control de Infecciones (MINSAL-IIH), recopila la información de resistencia bacteriana enviada durante 2 meses del año (un mes del primer semestre y un mes durante el segundo semestre) en 58 hospitales del país y corresponde a una prevalencia en patógenos de importancia nosocomial: Staphylococcus aureus resistente a oxacilina, Klebsiella pneumoniae, Pseudomonas aeruginosa, Acinetobacter baumannii y Enterococcus sp resistente a vancomicina. A diferencia de la vigilancia de resistencia del IPS, esta vigilancia utiliza una metodología descentralizada (cada centro envía las cifras de resistencia, lo que permite una mejor cobertura), pero no tiene registros del control de calidad de cada laboratorio, por lo que no hay certificación de que las cifras obtenidas sean exactas y precisas.

- Red PRONARES: proyecto realizado por la

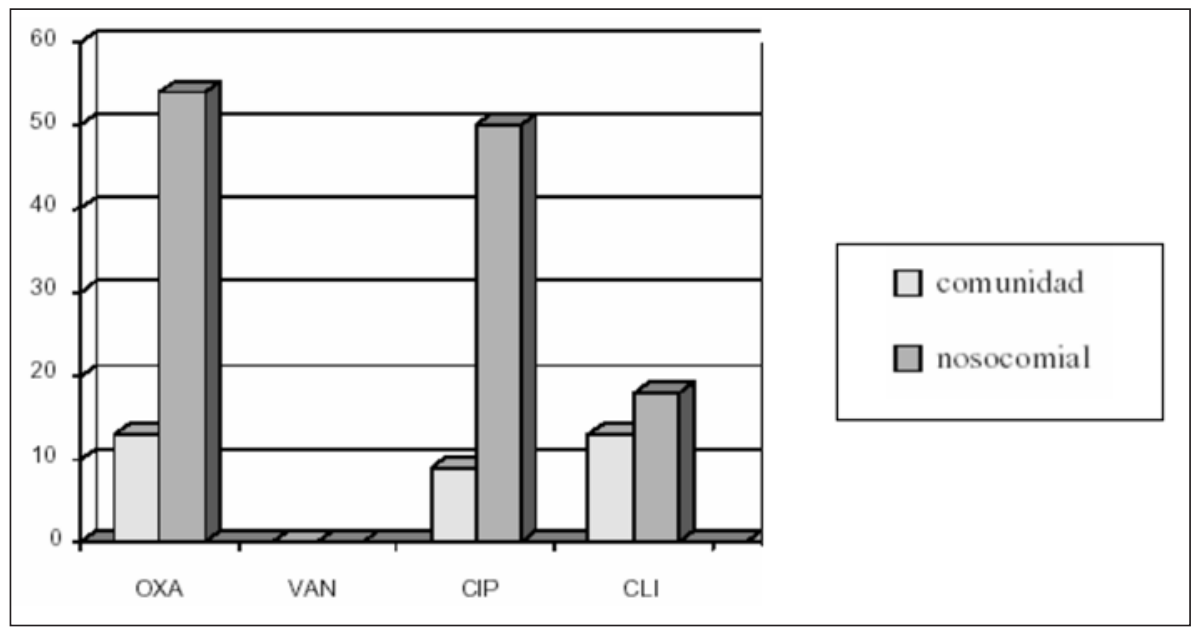

Grafico 2. Porcentaje de resistencia de $S$. aureus de la comunidad (241) y nosocomial (251) para oxacilina (OXA), vancomicina (VAN), ciprofloxacina (CIP) y clindamicina (CLI). Fuente: Pronares; Trucco et al. Rev Chil Infec 2002; 19: S140-8. 
Tabla 2. Distribución de la resistencia de Streptococcus pneumoniae a penicilina según grupo etario

\begin{tabular}{|c|c|c|c|c|}
\hline \multirow[t]{2}{*}{ Grupo etario } & \multirow{2}{*}{$\begin{array}{c}\text { CIM } \\
\mathrm{S} \\
\leq 0,06\end{array}$} & \multicolumn{2}{|c|}{ Penicilina $(\mu \mathrm{g} / \mathrm{ml})$} & \multirow{2}{*}{$\begin{array}{c}\text { Total } \\
\text { cepas estudiadas }\end{array}$} \\
\hline & & $\begin{array}{c}\mathrm{I} \\
0,12-1\end{array}$ & $\begin{array}{c}\mathrm{R} \\
\geq 2\end{array}$ & \\
\hline$<5$ años & 72,8 & 14,3 & 12,8 & 530 \\
\hline$>5-<15$ años & 83,7 & 8,7 & 7,6 & 92 \\
\hline $15-60$ & 91,6 & 4,8 & 3,6 & 167 \\
\hline$>60$ años & 97,0 & 1,5 & 1,5 & 67 \\
\hline
\end{tabular}

Fuente: Laboratorio de Referencia, Instituto de Salud Pública de Chile.

Universidad de Chile para lograr una información de resistencia bacteriana en relación al sitio de aislamiento bacteriano y a la procedencia de las muestras (intra o extrahospitalarias), que ha permitido disponer de información muy útil para algunos patógenos no vigilados por el ISP. El proyecto contempló la implementación del control de calidad; sin embrago, sólo participaron 5 hospitales de regiones distintas de la Metropolitana.

- Datos obtenidos de grandes programas de vigilancia mundial como SENTRY, coordinado por la Universidad de Iowa, E.U.A., que exige el envío mensual de las cepas de infecciones definidas, en un número representativo, pero que en nuestro país sólo cuenta con la participación de dos centros nacionales, lo que disminuye la representatividad de las cifras, considerando que los centros partici- pantes son hospitales de alta complejidad.

- Datos locales publicados en la Revista Chilena de Infectología o en la Revista Médica de Chile, en que los resultados representan estudios bien realizados, pero que sólo representan la realidad local del centro desde donde se obtienen los datos.

- Datos obtenidos por encuestas realizadas a algunos centros, en donde generalmente no hay ni representatividad ni un buen registro del control de calidad.

En definitiva, la estimación de la magnitud del problema de la resistencia bacteriana en Chile tiende a ser un promedio de la información obtenida por estas diversas fuentes, quedando en absoluta evidencia la necesidad de una Red

Tabla 3. Comparación de la emergencia de la resistencia entre Estados Unidos de América y Chile a algunos agentes antimicrobianos seleccionados

\begin{tabular}{|c|c|c|c|c|c|}
\hline Microorganismo & Antimicrobiano & $\%$ resistencia $^{a}$ & $\uparrow \% / a \tilde{a n o}{ }^{b}$ & $\%$ resistencia $^{a}$ & $\uparrow \% / a \tilde{n} o^{b}$ \\
\hline & & \multicolumn{2}{|c|}{ E.U.A } & \multicolumn{2}{|c|}{ CHILE } \\
\hline S. aureus & Meticilina & $42,0 \%$ & $1,1 \%$ & $69 \%$ & $3,6 \%$ \\
\hline S. pneumoniae & Penicilina & $23,0 \%$ & $0,3 \%$ & $17 \%$ & $1,6 \%$ \\
\hline P. aeruginosa & Imipenem & $14,5 \%$ & $0,7 \%$ & - & - \\
\hline Enterococcus sp & Vancomicina & $11,0 \%$ & $0,6 \%$ & $18 \%$ & - \\
\hline K. pneumoniae & Ceftazidima $^{c}$ & $7,4 \%$ & $0,6 \%$ & $65 \%$ & $4,3 \%$ \\
\hline E. coli & Ciprofloxacina & $6,5 \%$ & $0,5 \%$ & - & - \\
\hline
\end{tabular}

Fuentes: EUA, C. Thornsberry. 103 General Meeting ASM 2003. Chile, Vigilancia IIH-MINSAL: Prevalencia mensual (abril y septiembre 1991-2001). Vigilancia ISP: Resistencia de S. pneumoniae en infecciones invasoras.

${ }^{\text {a }}$ El porcentaje de resistencia corresponde al año 2001.

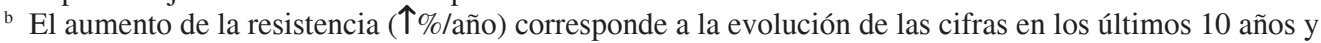
consiste en el aumento porcentual de la resistencia al año, que se considera como emergencia de la resistencia.

${ }^{c}$ En Chile se evalúa cefotaxima. 
Tabla 4: Mecanismos de resistencia descritos en Pseudomonas aeruginosa, que le confieren resistencia a los antimicrobianos.

\begin{tabular}{ll}
\hline Mecanismo de resistencia & Antimicrobiano al cual es resistente \\
\hline$\beta$-lactamasas & $\beta$-lactámicos \\
$\beta$-lactamasas de espectro extendido & Cefalosporinas de amplio espectro \\
Carbapenemasas, alteración en las porinas & Carbapenémicos \\
Enzimas modificantes de aminoglucósidos & Aminoglucósidos \\
Alteración de ADN girasa & Quinolonas \\
Bombas de eflujo & Se han descrito para todos los AAM \\
\hline
\end{tabular}

Nacional de Resistencia que permita obtener datos en forma fidedigna, representativa, por síndromes clínicos y según procedencia de los pacientes.

\section{Complejidad de la resistencia bacteriana}

Por otro lado, el problema de la resistencia bacteriana es complejo (Figura 2) y requiere considerar:

- Factores relacionados al paciente: es diferente según la edad del paciente (niño/adulto), según la procedencia del paciente (ambulatorio/ hospitalizado), según el síndrome clínico o localización de la infección o según si es infección significativa o colonización. Respecto de la edad del paciente, en la Tabla 2, se observa que a menor edad, existe una mayor probabilidad de adquirir una infección por Streptococcus. pneumoniae resistente (datos ISP Chile, no publicados). Respecto de la procedencia del paciente, en el informe de PRONARES del año 2001 (Gráfico 1 y 2) se observa una clara diferencia en todos los AAM evaluados para Klebsiella sp y Staphylococcus aureus según sean infecciones de la comunidad o de adquisición nosocomial ${ }^{2}$.

- Factores relacionados al microorganismo: es importante considerar el tipo de microorganismo, la resistencia múltiple y la emergencia de la resistencia.

Respecto del tipo de microorganismo es importante tener en cuenta que para un mismo AAM, como por ejemplo penicilina, existe importante variación en la resistencia en el grupo de cocáceas Gram positivas: durante el año 2001-
2002 S. aureus presentó un $90 \%$ de resistencia versus $17 \%$ en $S$. pneumoniae y $0 \%$ en Streptococcus pyogenes ${ }^{2,3}$. Datos ISP no publicados). En el caso de ciprofloxacina sobre bacilos Gram negativos, se observa también variabilidad entre Escherichia coli con 5 a $15 \%$ de resistencia y Pseudomonas aeruginosa con 30 a $35 \%$ de resistencia ${ }^{2,3}$.

Respecto de la resistencia múltiple y emergencia de la resistencia, ambos se consideran problemas de gran impacto en la actualidad.

En la Tabla 3 se muestran los aumentos porcentuales en la resistencia de algunos microorganismos de relevancia médica en E.U.A. ${ }^{4}$. El aumento del porcentaje de resistencia se conoce como emergencia de la resistencia asumiéndose que cuando el AAM es introducido al mercado el porcentaje de resistencia es cercano a cero. El conocer estos datos permitirá estimar las expectativas de vida útil de una AAM. Utilizando los datos del Ministerio de Salud en infecciones intrahospitalarias y los de la vigilancia de resistencia de infecciones invasoras por S. pneumoniae, que registran cifras de los 10 últimos años (19912001), se puede estimar que la emergencia de la resistencia de $K$. pneumoniae a cefotaxima es de 4,3\% anual, de $S$. aureus a oxacilina de 3,6\% anual y de $S$. pneumoniae a penicilina de $1,6 \%$ anual. Estos aumentos en los porcentajes de resistencia por año son mayores que los observados para E.U.A. y son preocupantes respecto de las expectativas de vida útil de oxacilina para $S$. aureus y cefotaxima para $K$. pneumoniae. No se disponen datos de resistencia en $P$. aeruginosa a imipenem.

Este último problema junto con la resistencia en $E$. coli y $K$. pneumoniae a las cefalosporinas 


\section{Porcentaje de susceptibilidad}

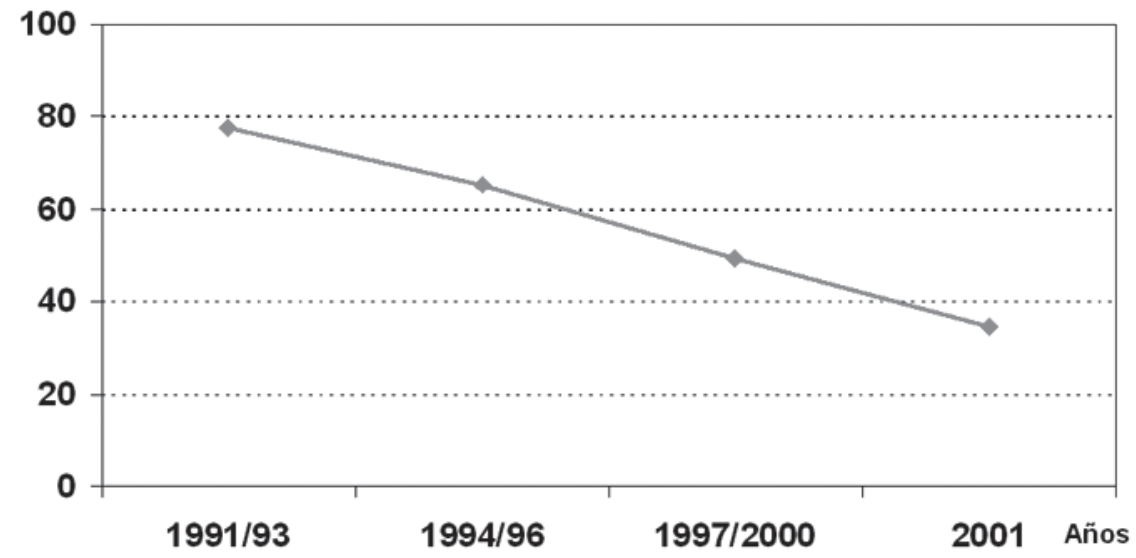

cepas estudiadas:

1385

Gráfico 3. Evolución de la susceptibilidad de K. pneumoniae a cefotaxima en Chile entre los años 1991 y 2001. Fuente: Minsal - IIH: Vigilancia de la resistencia en patógenos hospitalarios. Datos no publicados.

de tercera generación por aparición de cepas productoras de $\beta$-lactamasas de espectro extendido (BLEE), serán analizados con más detalle.

- Factores relacionados al fármaco: se debe considerar el modo de empleo de un determinado agente antimicrobiano, las dosis utilizadas, la vía de administración y la duración de la terapia. También se debe considerar el espectro del AAM que se está utilizando (amplio o reducido). Todas estas variables inciden en el desarrollo o selección de la resistencia.

\section{Resistencia bacteriana emergente en Chile}

\section{Resistencia en Pseudomonas aeruginosa}

Esta bacteria es un importante patógeno nosocomial, especialmente en infecciones respiratorias bajas asociadas a ventilación mecánica, infecciones asociadas a catéter urinario, infecciones de sitio quirúrgico e infecciones del torrente sanguíneo 5 .

Posee características fisiológicas que le permiten ser un patógeno oportunista efectivo: mínimos requerimientos nutricionales, tolerancia a condiciones físicas extremas y la capacidad de desarrollar rápidamente resistencia a los antimicrobianos. En la Tabla 4 se muestran los mecanismos de resistencia que se han demostrado en $P$. aeruginosa para $\beta$-lactámicos incluyen- do carbapemens, aminoglucósidos y fluoroquinolonas. Si bien se han descrito $\beta$-lactamasas comunes, disminución de la permeabilidad a $\beta$ lactámicos, carbapenemasas, BLEE, enzimas modificantes de aminoglucósidos, alteración en la ADN girasa, la presencia de bombas de eflujo jugarían el rol más importante en la resistencia intrínseca y adquirida de $P$. aeruginosa.

La vigilancia de la resistencia centralizada (derivación de las cepas a tres laboratorios de referencia) a través del programa SENTRY (Tabla 5) en 6.631 cepas de P.aeruginosa aisladas desde 1997 a 1999 en América latina, Asia-Pacífico, E.U.A., Canadá y Europa, muestra una importante variación en las distintas áreas, observándose las cifras más altas de resistencia en América latina y Asia-Pacífico con un marcado aumento anual en el porcentaje de cepas multiresistentes, lo que se asocia con altas tasas de morbilidad y mortalidad $^{6}$. Esta marcada diferencia en las tasas de resistencia puede ser explicada probablemente por las diferencias en las prácticas en el uso de AAM y las diferencias en las prácticas de control de infecciones. También se puede inferir de la evolución dentro de estos tres años, que las tasas de resistencia continuarán en aumento a menos que se tomen las medidas adecuadas. Por esto resulta esencial la vigilancia activa de la resistencia para poder elaborar guías efectivas de terapia antimicrobiana. En un intento por disponer de cifras nacionales se muestran los datos de sus- 


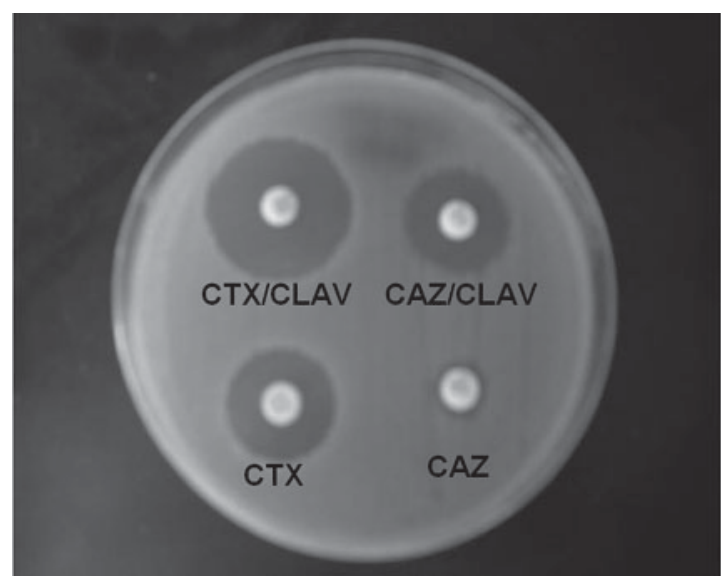

Figura 3. Método confirmatorio (NCCLS), para la presencia de BLEE. Aumento de $5 \mathrm{~mm}$ en el halo de inhibición si se utiliza un disco con la combinación cefalosporina + ácido clavulánico en relación a cuando se usa un disco solo con la cefalosporina. CTX = cefotaxima (30 $\mu$ g) CTX/ CLAV = cefotaxima $(30 \mu \mathrm{g})$ con ácido clavulánico $(10 \mu \mathrm{g})$ $\mathrm{CAZ}=$ ceftazima $(30 \mu \mathrm{g}) \mathrm{CAZ} / \mathrm{CLAV}=$ ceftazima $(30 \mu \mathrm{g})$ con ácido clavulánico $(10 \mu \mathrm{g})$

ceptibilidad en este microorganismo obtenidas desde la vigilancia de resistencia mediante el envío de datos correspondientes a los meses de abril/septiembre de cada año. En el Gráfico 3 se muestra la evolución de la susceptibilidad de $P$. aeruginosa a ceftazidima y gentamicina, en donde se aprecia que los porcentajes se han mantenido constantes en los últimos 10 años. Cuando se compara la información obtenida de las distintas fuentes durante los años 1999-2001 y se compara la estimación con los datos del SENTRY en América Latina, se observa que para ceftazidima, cefepime, imipenem y amikacina, las cifras son coincidentes desde datos obtenidos del SENTRY en Chile, los datos obtenidos en la red PRONARES y los datos del MINSAL-Infecciones intrahospitalarias, siendo los porcentajes de susceptibilidad en Chile mayores que los observados para América Latina. Para ceftazidima la susceptibilidad de $P$. aeruginosa es alrededor de $80 \%$, para cefepime alrededor de $90 \%$, para amikacina de $85 \%$ y para imipenem de $95 \%$. Existe más dispersión en los datos obtenidos en gentamicina y ciprofloxacina en que los porcentajes varían entre 56 y $73 \%$ y 67 a $82 \%$, respectivamente.

\section{Problemas técnicos con la determinación de susceptibilidad a imipenem en Pseudomonas aeruginosa}

En la determinación de la susceptibilidad de $P$. aeruginosa a imipenem es necesario destacar que este es un fármaco inestable ${ }^{7,8}$, que pierde activi-

Tabla 5. Porcentaje de susceptibilidad de Pseudomonas aeruginosa por Región desde 1997 a 1999. Programa SENTRY de Vigilancia de Resistencia ${ }^{5}$

\begin{tabular}{|c|c|c|c|c|c|c|c|c|c|c|c|c|c|c|}
\hline \multirow[b]{3}{*}{ Antimicrobial agent } & \multicolumn{14}{|c|}{ Percentage of isolates susceptible ${ }^{a}$} \\
\hline & \multicolumn{2}{|c|}{ Asia-Pacilic } & \multicolumn{3}{|c|}{ Canadá } & \multicolumn{3}{|c|}{ Europa } & \multicolumn{3}{|c|}{ América Latina } & \multicolumn{3}{|c|}{ E. U. A. } \\
\hline & 1998 & 1999 & 1997 & 1998 & 1989 & 1997 & 1998 & 1999 & 1997 & 1998 & 1999 & 1997 & 1998 & 1998 \\
\hline \multicolumn{15}{|l|}{ B-Lactams } \\
\hline Aztreonam & 66.5 & 65.3 & 73.4 & 67.1 & 75.2 & 80.2 & 73.2 & $55.6^{a}$ & 55.5 & 45.0 & 48.2 & 67.0 & 64.7 & 62.3 \\
\hline Pipera cilina & 85.5 & 85.8 & 91.7 & 87.4 & 89.1 & 85.6 & 77.0 & $73 . \mathrm{B}^{\mathrm{b}}$ & 71.9 & 66.7 & 67.5 & 87.9 & 87.3 & $83.7^{2}$ \\
\hline Piperacillinftazobactam & 90.2 & 87.5 & 93.2 & 90.4 & 95.6 & 90.1 & 77.0 & $73 . \mathrm{B}^{\mathrm{b}}$ & 79.4 & 77.1 & 74.9 & 89.9 & 89.9 & $86.6^{a}$ \\
\hline Ceftazidime & 76.1 & $83.7^{\text {d }}$ & 80.2 & 80.2 & 84.7 & 85.1 & 78.2 & $71.6^{\mathrm{a}}$ & 66.6 & 64.4 & 66.9 & 79.5 & 81.2 & 78.1 \\
\hline Cefepime & 81.6 & 85.8 & 80.2 & 84.4 & $91.2^{\circ}$ & 80.8 & 83.0 & $73.8^{\circ}$ & 66.2 & 67.9 & 66.3 & 77.7 & 85.8 & $83.1^{\mathrm{D}}$ \\
\hline Imipenem & 90.0 & 86.2 & 83.0 & 82.0 & $92.0^{\circ}$ & 89.3 & 79.0 & $71.6^{\mathrm{a}}$ & 77.0 & 76.7 & 74.3 & 88.0 & 85.2 & 80.9 \\
\hline Meropenem & 91.5 & 87.8 & 92.4 & 91.6 & 94.9 & 89.8 & 85.6 & $73 . \mathrm{B}^{\mathrm{a}}$ & 83.0 & 79.7 & $76.6^{\mathrm{b}}$ & 92.4 & 90.8 & 90.9 \\
\hline \multicolumn{15}{|l|}{ Aminoglucósidos } \\
\hline Amikacina & 94.2 & 95.8 & 91.4 & 95.8 & $97.8^{\circ}$ & 89.0 & 86.8 & $78.9^{\mathrm{C}}$ & 77.6 & 73.3 & $69.5^{\circ}$ & 95.0 & 94.8 & 96.6 \\
\hline Tobramicina & 88.5 & 89.9 & 91.4 & 93.4 & 94.2 & 76.3 & 78.3 & $68.4^{c}$ & 68.1 & 64.9 & 64.2 & 91.1 & 92.7 & 92.2 \\
\hline \multicolumn{15}{|l|}{ Quinolonas } \\
\hline Ciprofloxacina & 84.9 & 83.7 & 79.9 & 74.3 & 81.0 & 73.5 & 73.8 & 67.6 & 67.2 & 60.8 & 60.9 & 79.8 & 77.9 & 75.36 \\
\hline Levofloxacina & 83.9 & 82.6 & 74.5 & 70.1 & 80.3 & 71.1 & 73.4 & 68.4 & 63.6 & 59.0 & 59.5 & 74.4 & 73.7 & 73.4 \\
\hline Gatifloxacina & 80.1 & 77.5 & 66.2 & 64.1 & 74.5 & 72.0 & 72.2 & 64.0 & 60.9 & 57.1 & 56.8 & 69.1 & 69.0 & 67.2 \\
\hline Trovafloxacina & 84.8 & 82.0 & 76.6 & 74.1 & 81.0 & 70.6 & 73.9 & 68.4 & 65.4 & 60.1 & 60.7 & 75.2 & 77.4 & 73.7 \\
\hline Tetraciclina & 2.6 & 4.2 & 2.9 & 3.0 & 2.9 & 6.8 & 4.7 & $2.2^{\mathrm{c}}$ & 1.2 & 1.7 & 1.8 & 2.7 & 2.3 & 3.5 \\
\hline
\end{tabular}

Fuente: Gales AC; Jones RN; Turnidge J; Rennie R; Ramphal R. Characterization of Pseudomonas aeruginosa isolates: Occurrence rates, antimicrobial susceptibility patterns and molecular typing in the Global SENTRY an Antimicrobial Surveillance Program, 1997-1999. CID 2001; 32:S146-S155. 
dad si es almacenado a temperaturas mayores de $4^{\circ} \mathrm{C}$. Se han descrito varios pseudobrotes de $P$. aeruginosa resistente a imipenem en laboratorios usuarios de sistemas automatizados ${ }^{9-12}$, a pesar de un buen control de calidad. En uno de los centros afectados ${ }^{11}$ hubo un aumento en la tasa de $P$. aeruginosa resistente a imipenem desde $12 \%$ histórico a $23 \%$, que no resultaron ser epidemiológicamente relacionados ni clonales por electroforesis en campo pulsado, por lo que se dudó de los estudios de susceptibilidad; al chequearse por otro método, las cepas resultaron ser susceptibles. La determinación por cromatografía líquida de alta resolución (HPLC) de la concentración de imipenem en los paneles automatizados mostró una concentración de fármaco activo menor que la esperada, confirmando la degradación del antimicrobiano probablemente por un problema en el almacenamiento. Este pseudobrote por fallas técnicas no pudo ser detectado a pesar de un adecuado control de calidad con la cepa control $P$. aeruginosa ATCC 27853 , ya que esta cepa es extremadamente susceptible a imipenem y las inactivaciones parciales en el fármaco no son suficientes para dejar de inhibir el desarrollo de la cepa control.

Basados en esta publicación y en una posterior del grupo de calidad y validación de los tests de susceptibilidad por parte del CDC en Atlanta ${ }^{13}$, en que observó que sólo 74\% (241/325) de la cepas de $P$. aeruginosa derivadas como resistentes eran efectivamente resistentes, se ha establecido que las resistencias a imipenem deberían ser confirmadas por un método alternativo, si se observa un aumento inusual de las cifras de resistencia ${ }^{14}$.

\section{Resistencia por $\beta$-lactamasas de espectro extendido (BLEE)}

Estas son enzimas que hidrolizan todos los $\beta$ lactámicos (los oximino- $\beta$-lactámicos) incluyendo aztreonam, por lo cual una cepa productora de BLEE, se debe considerar resistente a todos los $\beta$-lactámicos. Son el resultado de mutaciones puntuales que ocasionan cambio en un aminoácido en las clásicas $\beta$-lactamasas TEM y SHV. A la fecha se han descrito más de 90 BLEE derivadas de la familia TEM y más de 25 BLEE derivadas

Tabla 6. Porcentaje de susceptibilidad de Pseudomonas aeruginosa en Chile según diferentes fuentes de información, según año, número de cepas y localización de la infección

\begin{tabular}{|c|c|c|c|c|c|}
\hline \multicolumn{6}{|c|}{ Porcentaje de susceptibilidad (\%) } \\
\hline $\begin{array}{l}\text { Fuente } \\
\text { Año } \\
\mathbf{N}^{\circ} \text { cepas } \\
\text { Localización }\end{array}$ & $\begin{array}{c}\text { SENTRY AL } \\
1999 \\
478 \\
\text { Todas }\end{array}$ & $\begin{array}{c}\text { SENTRY CH } \\
\text { 1997-2000 } \\
78 \\
\text { Invasoras }\end{array}$ & $\begin{array}{c}\text { PRONARES } \\
2001 \\
45 \\
\text { Invasoras }\end{array}$ & $\begin{array}{c}\text { MINSAL-IIH } \\
2001 \\
251 \\
\text { Todas }\end{array}$ & $\begin{array}{c}\text { Encuesta } \\
2003 \\
187 \\
\text { Todas }\end{array}$ \\
\hline Aztreonam & 48 & - & - & - & - \\
\hline $\begin{array}{l}\text { Piperacilina / } \\
\text { tazobactam }\end{array}$ & 75 & 82 & - & - & - \\
\hline Ceftazidima & 67 & 80 & 82 & 82 & 81 \\
\hline Cefepime & 66 & 91 & 95 & - & - \\
\hline Imipenem & 74 & 97 & 91 & - & 90 \\
\hline Meropenem & 77 & 97 & - & - & - \\
\hline Amikacina & 70 & 89 & 88 & 81 & 80 \\
\hline Gentamicina & - & - & 73 & 56 & - \\
\hline Ciprofloxacina & 61 & 82 & 69 & 67 & 60 \\
\hline
\end{tabular}

Fuente: SENTRY AL América Latina (Gales et al. CID 2001; 32:S147-S155), SENTRY Chile (Sader et al. Diagn Microbiol Infect Dis 2003; 44: 273-280), PRONARES (Trucco et al. Rev Chil Infect 2002;19: S140-148), IIH MINSAL (Vigilancia de la Resistencia en Patógenos hospitalarios), Encuesta a 11 Laboratorios de Chile. 
Tabla 7: Métodos recomendados por NCCLS para la sospecha fenotípica de cepas de $E$. coli, $K$. pneumoniae y $K$. oxytoca productoras de BLEE

\begin{tabular}{lccc}
\hline \multicolumn{2}{c}{ Para difusión en disco } & \multicolumn{2}{c}{ Para métodos CIM* } \\
\hline $\begin{array}{ccc}\text { Antimicrobiano } \\
\end{array}$ & $\begin{array}{c}\text { Halo } \\
\text { inhibición } \\
(\mathbf{m m})\end{array}$ & Antimicrobiano & $\begin{array}{c}\text { CIM* } \\
(\boldsymbol{\mu g} / \mathbf{m l})\end{array}$ \\
& $\leq 17 \mathrm{~mm}$ & Cefpodoxima & $\geq 4 \mu \mathrm{g} / \mathrm{ml}$ \\
Cefpodoxima & $\leq 22 \mathrm{~mm}$ & Ceftazidima & $\geq 2 \mu \mathrm{g} / \mathrm{ml}$ \\
Ceftazidima & $\leq 27 \mathrm{~mm}$ & Aztreonam & $\geq 2 \mu \mathrm{g} / \mathrm{ml}$ \\
Aztreonam & $\leq 27 \mathrm{~mm}$ & Cefotaxima & $\geq 2 \mu \mathrm{g} / \mathrm{ml}$ \\
Cefotaxima & $\leq 25 \mathrm{~mm}$ & Ceftriaxona & $\geq 2 \mu \mathrm{g} / \mathrm{ml}$ \\
Ceftriaxona & $\leq$ &
\end{tabular}

*Concentración Inhibitoria Mínima.

de la familia SHV. Estas se han descrito principalmente en E. coli, K. pneumoniae y $K$. oxytoca, pero también se han descrito BLEE en Salmonella sp (CTX-M) y en $P$. aeruginosa (PER-1 y VEB1). Funcionalmente la modificación en un aminoácido producen un cambio en el punto isoeléctrico de la enzima y la capacidad de hidrolizar las cefalosporinas de tercera generación y aztreonam (oximino $\beta$-lactámicos); sin embargo, la expansión del sitio activo de la enzima también le confiere aumento en la susceptibilidad a los inhibidores de $\beta$-lactamasas ${ }^{15}$.

La detección de este tipo de enzimas es muy importante en el laboratorio, ya que la presencia de BLEE confiere resistencia a todos los $\beta$ lactámicos incluyendo aztreonam, aunque sean susceptibles in vitro. Clínicamente se ha asociado con un aumento de la estadía hospitalaria y mayor costo en antimicrobianos ${ }^{16}$.

\section{Determinación de $\boldsymbol{\beta}$-lactamasas de espectro extendido en Escherichia coli y Klebsiella pneumoniae}

Si bien la presencia de una BLEE le confiere a la bacteria resistencia a uno o más oximino $\beta$ lactámicos, no siempre producen un aumento de la CIM como para ser clasificada como resistente. Esto ocasiona problemas con la sensibilidad de la detección de BLEE, lo que es muy importante desde el punto de vista clínico, ya que independiente de los resultados in vitro, la detección de BLEE lleva a informar como resistentes todas las cefalosporinas de tercera generación.

A la fecha no se ha descrito ningún método de laboratorio que sea $100 \%$ sensible ni $100 \%$ específico en la detección de BLEE y en general, los métodos microbiológicos dependen de la cefalosporina utilizada para realizar los tests de susceptibilidad $^{17}$.

Las recomendaciones para los laboratorios en E.U.A. (NCCLS) para la sospecha y confirmación de BLEE se observan en la Tabla 7.

Los laboratorios de microbiología deben sospechar que una cepa de E. coli, K. pneumoniae o $K$. oxytoca es productora de BLEE cuando de observe una reducción en los halos de inhibición o un aumento en las CIM para cefalosporinas de tercera generación, sin llegar a ser considerados dentro de la categoría resistente, lo que significa una especial atención de los laboratorios en la sospecha de $\mathrm{BLEE}^{7}$.

Los métodos confirmatorios fenotípicos se basan en la capacidad del ácido clavulánico para inhibir las $\beta$-lactamasas, de modo que para los métodos CIM se considera confirmatorio una disminución de la CIM en tres o más diluciones cuando se evalúa cefotaxima sola versus cefotaxima con ácido clavulánico o ceftazidima sola versus ceftazidima con ácido clavulánico. Del mismo modo para la difusión en agar se considera confirmatorio un aumento de $\geq 5 \mathrm{~mm}$ en el halo de inhibición cuando se evalúa cefotaxima sola versus cefotaxima con ácido clavulánico o ceftazidima sola versus ceftazidima con ácido clavulánico (Figura 3). Se han buscado alternativas de más bajo costo, que no requieran utilizar cuatro discos para el test confirmatorio (por ejemplo el uso de sólo un par de discos: cefpodoxima sola y cefpodoxima con ácido clavulánico); sin embargo, la sensibilidad del método disminuye ${ }^{18}$. También de han descritos otros métodos confirmatorios fenotípicos como el Etest ESBL (AB Biodisk, Solna, Sweden) en que la tira está impregnada por un extremo sólo con ceftazidima y en el otro extremo con ceftazidima más ácido clavulánico ${ }^{19}$, o los sistemas automatizados con tarjetas confirmatorias como Vitek (Biomerieux, Hazelwood, Mo) que tienen pocillos con la cefalosporina sola y otro con la cefalosporina más ácido clavulánico ${ }^{20}$. En general estos métodos son fáciles de usar, pero probablemente muy caros para ser utilizados en la rutina en nuestro país.

Por lo común los laboratorios clínicos fallan en sospechar la presencia de BLEE o en informar la sospecha en el resultado del estudio de susceptibilidad.

En un estudio reciente conducido por la OMS sólo 2 de 130 laboratorios participantes informaron los resultados de susceptibilidad de Klebsiella sp y E. coli como productores de $\mathrm{BLEE}^{21}$. En una encuesta contestada por 11 laboratorios en nuestro país (7 de regiones y 4 de Santiago), sólo 2 de ellos realizaban tests confirmatorios para 


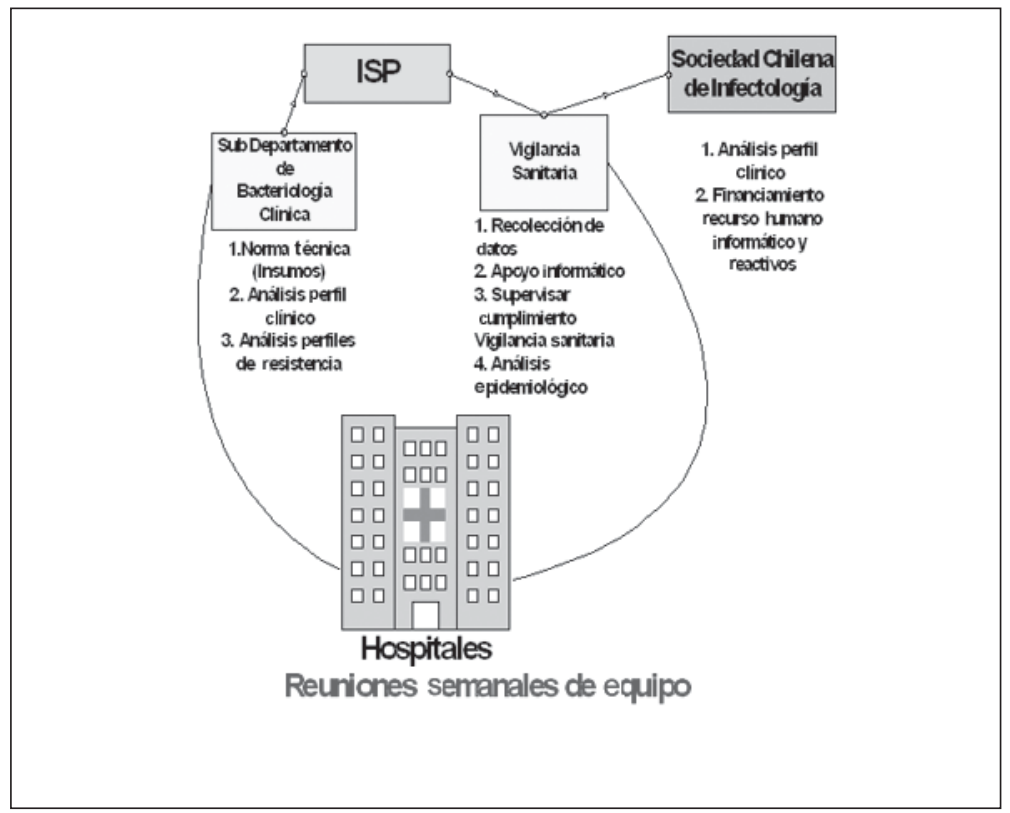

Figura 4. Esquema del funcionamiento de la Red Nacional de Resistencia, con la participación del Instituto de Salud Pública, la Sociedad Chilena de Infectología y los hospitales de los Servicios de Salud.

cepas sospechosas de BLEE y sólo 5 laboratorios sospechaban la presencia de BLEE por la reducción de halos y agregan una nota al informe advirtiendo al médico clínico de la probable presencia de BLEE.

Si no existe sospecha frente a la disminución de halos de inhibición, y se espera una resistencia a cefalosporinas de tercera para confirmar la presencia de BLEE en una cepa, sólo se estará pesquisando un porcentaje de las cepas productoras. Asumiendo que la mayoría de las cepas de E. coli y $K$. pneumoniae resistentes a cefalos-porinas de tercera generación son productoras de $\mathrm{BLEE}^{22,23}$, se puede inferir a partir de los datos entregados por el MINSAL-Infecciones intra-hospitalarias, que desde el año 1991 al 2001, la proporción de cepas productoras de BLEE en $K$. pneumoniae ha aumentado en forma importante ya que la susceptibilidad a cefotaxima en las cepas de origen hospitalario ha disminuido desde casi $80 \%$ en 1991 a 35\% en el año 2001 (Gráfico $3)$.

Los datos de Mella y colaboradores ${ }^{24}$ muestran que $43,9 \%$ de $K$ pneumoniae y $8 \%$ de $E$. coli son productoras de BLEE, cifras coincidentes con los publicados para Chile por Sader et al en que $49,6 \%$ de $K$ pneumoniae y $7,6 \%$ de $E$. coli aisladas de infecciones del torrente sanguíneo fueron productoras de BLEE ${ }^{3}$.

\section{Obtención de datos fidedignos: Red Nacional de Resistencia}

Como se muestra en la Figura 1, el problema de la resistencia bacteriana es complejo, multifactorial, cuya contención requiere de medidas que ya se están implementando en nuestro país; sin embargo, la estimación de la magnitud de la resistencia en nuestro país ha sido un problema no resuelto. En el año 2002 la Sociedad Chilena de Infectología en conjunto con el Instituto de Salud Pública de Chile decidieron iniciar un desafío común: implementar una Red Nacional para la Vigilancia de la Resistencia a los Antimicrobianos. Los objetivos de esta red son obtener datos de resistencia confiables y representativos del país, que nos permitan conocer la resistencia por síndrome clínico, diferenciando niños de adultos, infecciones comunitarias de las nosocomiales y teniendo como requisitos una buena calidad de las muestras y un buen control de calidad por parte del laboratorio de microbiología. El documento que define la implementación de la Red fue recientemente publicado ${ }^{25}$, así como una nota editorial de la Presidenta de la Sociedad Chilena de Infectología respecto de la importancia de la red ${ }^{26}$. El proyecto comenzó a funcionar el $1^{\circ}$ de abril del 2003 con la participación de 42 centros de 47 convocados, y una modalidad de funcionamiento que se muestra en la Figura 4. 
Los laboratorios de los hospitales locales deben completar una información normalizada en una planilla de ingreso de los datos por síndrome clínico, por edad y por tipo de infección (nosocomial/comunitaria). Para cada síndrome están definidos los antimicrobianos que deben ensayarse y está definida la frecuencia del control de calidad para los estudios de susceptibilidad. El ingreso de los datos se realiza en el sistema WHONET, diseñado por la OMS para el ingreso computacional de datos de resistencia de acuerdo a las normas NCCLS ${ }^{7}$.

A la fecha se han visitado la totalidad de los centros a lo largo del país y se estima que para noviembre del presente año se dispondrá de información preliminar de resistencia a antimicrobianos de infecciones invasoras y del tracto respiratorio superior e inferior.

\section{Conclusiones}

Es necesario destacar que dado que el problema de la resistencia bacteriana requiere un trabajo en equipo para lograr su control, resulta necesario:

- Disponer de cifras nacionales confiables a través de una Red Nacional de Vigilancia de la Resistencia que nos permita estimar la magnitud del problema.

- Continuar con la restricción en el uso de antimicrobianos iniciada en nuestro país en septiembre de 1999 por iniciativa de la Sociedad Chilena de Infectología.

- Evitar el abuso de antimicrobianos en su prescripción individual.

- Robustecer los programas de control de infecciones intrahospitalarias.

- Disponer de laboratorios de Microbiología adecuados, confiables capaces satisfacer las necesidades de los pacientes, siendo especialmente importante la certificación de la calidad de los resultados.

- Evaluar la emergencia de la resistencia bacteriana que nos permitirá como país estimar las expectativas de vida útil de los antimicrobianos, para disponer de guías clínicas de tratamiento.

- Identificar bacterias que diseminan la resistencia con mayor velocidad.

- Continuar con la investigación en el ámbito nacional.

Agradecimientos

La obtención de los datos fue posible gracias :

Ministerio de Salud: Fernando Otaíza O’R y Patricio Nercelles M

Instituto de Salud Pública de Chile: Andrea
Sakurada Z. Soledad Prat M. Bernardita Monje A.

Sociedad Chilena de Infectología: M. Teresa Valenzuela B y Olivia Trucco A.

Participantes de la Encuesta: Paola Gatica (H. Roberto Del Río), Beatrice Hervé (H. DIPRECA), Chrystal Juliet y Alejandra Fernández (H. Del Salvador), Mónica Lafourcade (H. San Juan de Dios), Fermín Méndez (H. Ernesto Torres de Iquique), Gerardo Peralta (H. Van Buren de Valparaíso), Víctor Cortés (H. Herminda Martin de Chillán), Marina Opazo (H. Guillermo Grant Benavente de Concepción), Maritza Navarrete ( $\mathrm{H}$. Clínico Regional de Valdivia), M. Luisa Rioseco (H Regional de Puerto Montt), Alicia González y Bernardo Vallejos (H. de Coyhaique)

\section{Resumen}

La resistencia bacteriana es un fenómeno complejo en que influyen factores como el uso y abuso de los antimicrobianos, el debilitamiento de los programas de control de infecciones y la existencia de pacientes complejos multi-invadidos. Dado que la resistencia bacteriana tiene como principal consecuencia el fracaso de la terapia antimicrobiana, el aumento de la morbimortalidad y el aumento en los costos de la atención médica, resulta indispensable la contención del problema. La etapa inicial de la contención es conocer la magnitud de la resistencia bacteriana, lo que en nuestro país ha resultado difícil por carecer de un sistema nacional de registro, unificado, de gran cobertura y con una calidad certificada. Con los datos disponibles a la fecha obtenidos de diversas fuentes de información, resulta preocupante la emergencia de resistencia en Staphylococcus aureus a meticilina, en Streptococcus pneumoniae a penicilina, en Escherichia coli y Klebsiella pneumoniae a cefalosporinas de tercera generación y de Pseudomonas aeruginosa a imipenem. En estos últimos aspectos es preocupante el aumento progresivo de resistencia de E. coli y $K$. pneumoniae a cefalosporinas de tercera generación por beta lactamasas de espectro extendido, que confieren resistencia a todos los beta lactámicos, cuyos test confirmatorios están escasamente implementados en nuestro país. Respecto de la resistencia de $P$. aeruginosa a carbapemémicos aún no es un problema de gran magnitud en Chile, pero en hospitales de alta complejidad deben existir terapias alternativas y métodos que permitan la confirmación de la resistencia a este antimicrobiano. En suma, queda manifiesta la absoluta necesidad de conocer en forma repre- 
sentativa y certificada las cifras de resistencia a los diferentes antimicrobianos, para adoptar las medidas terapeúticas necesarias.

\section{Bibliografía}

1.- File T M. Overview of resistance in the 1990s. Chest 1999; 115: S3-8.

2.- Trucco O, Prado V, Durán C y Grupo PRONARES. Red de vigilancia de resistencia antimicrobiana PRONARES. Informe primer semestre 2001. Rev Chil Infect 2002; 19: S140-8.

3.- Sader H, Jones R N, Andrade-Baiocchi S, Biedenbach D J and the SENTRY participant group (Latin América). Four-year evaluation of frecuency of occurrence and antimicrobial susceptibility patterns of bacteria from bloodstream infections in Latin American medical centers. Diag Microbiol Infect Dis 2003; 44: 273-80.

4.- Thornsberry C. Antimicrobial Resistance: Yesterday, today and tomorrow. 103 General Meeting American Society for Microbiology. Washington DC. May 2003.

5.- Gales A C, Jones R N, Turnidge J, Rennie R, Ramphal $\mathrm{R}$. Characterization of Pseudomonas aeruginosa isolates: Occurrence rates, antimicrobial susceptibility patterns and molecular typing in the Global SENTRY Antimicrobial Surveillance Program, 1997-1999. Clin Inf Dis 2001; 32: S146-55.

6.- Carmeli Y, Troillet N, Karchmer A W, Samore H M. Health and economic outcomes of antibiotic resistance in Pseudomonas aeruginosa. Arch Intern Med 1999; 159: $1127-32$.

7.- National Committee for Clinical Laboratory Standards. 2003. Methods for dilution antimicrobial susceptibility test for bacteria that grow aerobically; Approved Standard- Sixth Edition. M7A6, Vol 23 (2): 12.

8.- Grist R. External factors affecting imipenem performance in dried microdilution MIC plates. J Clin Microbiol 1992, 30: 535-6.

9.- O'Rourke E J, Lambert K G, Parsonnet K C, Macone A B, Goldmann D A. False resistance to imipenem with a microdilution susceptibility testing system. J Clin Microbiol 1991; 29: 827-9.

10.- White R L, Kays M B, Friedrich L V, Brown E W, Koonce J R. Pseudoresistance of Pseudomonas aeruginosa resulting from degradation of imipenem in an automated susceptibility testing system with predried panels. J Clin Microbiol 1991; 29: 398-400.

11.- Carmell Y, Eichelberger K, Soja D et al. Failure of quality control measures to prevent reporting of false resistance to imipenem in a pseudo-outbreak of imipenem-resistant Pseudomonas aeruginosa. J Clin Microbiol 1998; 36: 595-7.

12.- Joyanes P, del Carmen Conejo M, Martínez-Martínez, L, Perea E J. Evaluation of the VITEK 2 System for the identification and susceptibility testing of three species of nonfermenting Gram-negative rods frequently isolated from clinical samples. J Clin Microbiol 2001; 39: 3247-53.

13.- Steward C D, Mohammed J M, Swenson J M et al. Antimicrobial susceptibility testing of Carbapenems: Multicenter validity testing and accuracy levels of five antimicrobial test methods for detecting resistance in Enterobacteriaceae and Pseudomonas aeruginosa isolates. J Clin Microbiol 2003; 41: 351-8.

14.- Tennover F, Hindler J. CDC, Division of healthcare quality promotion, issue in healthcare settings. Laboratory detection of imipenem or meropenem resistance in Gram-negative organisms. http:// www.cdc.gov/ncidod/hip/Lab/FactSheet/imipenem.htlm

15.- Bradford P. Extended-Spectrum b-Lactamases in the $21^{\text {st }}$ Century: Characterization, epidemiology, and detection of this important resistance threat. Clin Microb Rev 2001; 14: 933-51.

16.- Lautenbach E, Patel J B, Bilker W B, Edelstein P H, Fishman N O. Extended-Spectrum b-Lactamase producing Escherichia coli and Klebsiella pneumoniae: Risk factors for infection and impact of resistance on outcomes. Clin Infect Dis 2001; 32: 1162-71.

17.- Jacoby G A, Han P. Detection of extended-spectrum $\beta$-lactamases in clinical isolates of Klebsiella pneumoniae and Escherichia coli. J Clin Microbiol 1996; 34: 908-11.

18.- Porte L, García P, Sánchez T, Varela C, Labarca J, Zamorano J. Estrategia de bajo costo para la confirmación fenotípica de $\beta$-lactamasas de espectro expandido. XIX Congreso Chileno de Infectología. 2001

19.- Cormican M G, Marshall S A, Jones R N. Detection of extended-spectrum $\beta$-lactamases-producing strains by the Etest ESBL screen. J Clin Microbiol 1996; 34: 1880-4.

20.- Sanders C C, Barry A L, Washington J A et al. Detection of extended-spectrum $\beta$-lactamasesproducing members of the Family Enterobacteriaceae with the Vitek ESBL Test. J Clin Microbiol 1996; 34: 2997-3001.

21.- Tenover F C, Mohamed M J, Stelling J, O’Brien T, Williams R. Ability of laboratories to detect emerging antimicrobial resistance: Proficiency testing and quality control results from the World Health Organization's External Quality Assurance System for antimicrobial susceptibility testing. J Clin Microbiol 2001; 39: 241-50.

22.- Sánchez T, García P, Riedel I, Guzmán A M. Evaluación de un sistema comercial para la detección de $\beta$ lactamasas de espectro expandido en cepas de E. coli y $K$. pneumoniae. XIX Congreso Chileno de Infectología. 2001.

23.- Zemelman R, Valenzuela L, Domínguez M, Bello H, González G, Zemelman C. Detección de $\beta$-lactamasas de espectro extendido en el laboratorio de microbiología. Rev Chil Infect 2002; 19 (Supl): S92-5.

24.- Mella S, Zemelman C, Bello H, Domínguez M, Gonzalez G, Zemelman R. Propiedades microbiológicas, clasificación y relación estructura-actividad de cefalosporinas e importancia de las cefalosporinas de cuarta generación. Rev Chil Infect 2001; 18: 7-19.

25.- Valenzuela M T, Prat S, Santolaya M E et al. Implementación de una Red Nacional para la Vigilancia de Resistencia de agentes patógenos a antimicrobianos según síndromes clínicos. Rev Chil Infect 2003; 20: 119-25.

26.- Santolaya ME. Implementación de una Red Nacional para la Vigilancia de Resistencia de agentes patógenos a antimicrobianos según síndromes clínicos. Editorial. Rev Chil Infect 2003; 20: 87-8. 